Please quote as: Köbler, F.; Koene, P.; Krcmar, H.; Altmann, M. \& Leimeister, J. M. (2010): LocaTag - An NFC-based system enhancing instant messaging tools with real-time user location. In: 2. International Workshop on Near Field Communication (NFC) 2010, Monaco, Monaco. 


\section{LocaTag - An NFC-based system enhancing instant messaging tools with real-time user location}

\author{
Felix Köbler, Philip Koene, Helmut Krcmar \\ Chair for Information Systems \\ Technische Universität München \\ Garching bei München, Germany \\ (felix.koebler | philip.koene | krcmar)@in.tum.de
}

\author{
Matthias Altmann, Jan Marco Leimeister \\ Chair for Information Systems \\ Universität Kassel \\ Kassel, Germany \\ (altmann | leimeister)@wi-kassel.de
}

\begin{abstract}
In recent years instant messaging tools have been successfully introduced to support communication and collaboration processes in work environments. Research suggests that the use of instant messaging tools lead to an increased feeling of connectedness, social presence and awareness within a collaborative group. Consequently, the goal of our research is to improve communication and collaboration within a group through automated and real-time dissemination of presence information, using instant messaging software. The paper describes LocaTag, a system that enhances instant messaging tools with real-time location information through the use of NFCenabled mobile devices. The LocaTag application is implemented as a prototype system that provides the Skype $^{\mathrm{TM}}$ instant messaging application with automated status messages, reflecting the current location of a user. This location information is derived from a NFC-tag based check-in/-out routine incorporating the mobile phone of the user. The tie-in of NFCbased location information and existing, broadly used instant messaging applications is an approach towards the real-time dissemination of presence information in collaborative groups. Its technical feasibility, limitations and future research approaches are discussed in this manuscript.
\end{abstract}

Keywords-Awareness systems, Instant messaging, Near Field Communication, Social presence

\section{INTRODUCTION}

$\mathrm{I}_{\mathrm{in}}^{\mathrm{n}}$ recent years advances in Internet technologies led to the implementation of functions generating a participative human-centric virtual environment. This environment enables communication and collaboration in large-scale online populations bundled in social networking platforms or synchronous and/or asynchronous text-based communication networks, e.g. Instant Messaging (IM) tools. Cameron and Webster [1] describe IM as a communication technology that allows users (e.g. employees) to send and receive short textbased messages or data files and to view the 'online' status, and thus availability of associates. IM is a low-cost communication and collaboration tool which is relatively easy to implement and use. Many popular IM systems are available for free distribution, including Yahoo! Messenger, Microsoft Network Messenger, ICQ, etc. Software vendors additionally provide enterprise versions of IM tools by targeting business customers, such as Effusia, Jabber, and Lotus Sametime, which are often integrated with other applications, e.g. workflow systems, and provide support functions beyond the scope of traditional IM tools.
The LocaTag prototype is based on the voice-over Internet Protocol (VoIP) application Skype ${ }^{\mathrm{TM}}$ which was primarily developed to enable telephone calls via the Internet, but additionally facilitates IM and awareness system functionalities, e.g. displaying presence information and usergenerated status messages. This specific type of message also prevalent in social networking platforms, i.e. Twitter and Facebook - serves as ad-hoc status reports with regards to a user's current emotional, situational and/or geographical state and is consecutively termed status message.

The paper presents the LocaTag prototype and describes different aspects of its design and development. The prototype allows a user to automatically set her Skype ${ }^{\mathrm{TM}}$ status message to the according location information stored on a NFC-tag by touching the tag with a NFC-enabled mobile device. We assume that the automated update of presence information leads to higher group awareness and therefore results in an improved communication and collaboration within a group [24].

The remainder of this paper is organized as follows: First, we like to outline a typical use case scenario and application environment of the LocaTag prototype. The following section describes the theoretical importance of connectedness, social presence and awareness in computer-supported communication and collaboration. The subsequent section outlines a definition of awareness systems and applications featuring awareness-conveying functionalities and summarizes related work. We then present a brief technical description of the LocaTag prototype application. The paper concludes with a discussion of potentials, limitations and future research prospects of the underlying concept.

\section{USE CASE SCENARIO}

In the following a use case scenario for a possible employment of the presented LocaTag prototype or similar applications is outlined:

John is working in a highly distributed software engineering and consulting project in which multiple teams are collocated between various geographical locations. Additionally, multiple teams are distributed between different parts of the building at the same site within the project environment Due to applied agile software development methods, the project teams switched from only virtual meetings to a mix of virtual and face-to-face meetings. This resulted in an increased travelling 
activity, not only by team managers but also by software developers, between different company and client facilities. In turn, previously introduced ad-hoc face-to-face meetings between team members from one location were highly efficient and productive because of the application of agile extreme programming methods, e.g. pair programming. The highly dynamic work environment significantly limited the possibility to conduct the essential ad-hoc meetings. The upper management identified two major causes: the unavailability of software developers due to geographical absence and the limited awareness of possible absence or availability of fellow software developers at the same site. John observed how his colleagues applied their instant messaging tool, not only for collaboration-related activities, but to inform fellow software developers of their current geographical status and/or location. In a next step, John called in a small team of fellow software developers and jointly proposed a technical concept for the automated and real-time dissemination of presence information using the company-wide distributed NFC-enabled mobile phones in combination with the corporate instant messaging tool.

A potential prototype that supports the described project environment is described in the scope of the underlying paper after an introduction to theory and related work found in Human Computer Interaction (HCI) and Computer Supported Cooperative Work (CSCW) literature.

\section{THEORETICAL BACKGROUND AND RELATED WORK}

\section{A. Theoretical importance of connectedness, social presence and awareness}

Connectedness, a concept being described in literature looking at communication and collaboration, can be described as the feeling of belonging to a social group and implies the creation of bonding relationships. IJsselsteijn et al. [5] hypothesize that for "this type of communication, the informational content of the message is of secondary importance to the emotional, relational content that is being transmitted". The concept of connectedness is related to concepts of social presence and awareness which have also been studied in previous research [6]. The difference between connectedness and social presence can best be illustrated referring to IM communication, as the awareness that peer users are online in the IM network conveys connectedness even when there is no message exchange. Rettie [6] argues that connectedness is a more fundamental concept, compared to social presence and awareness, that embodies a key concept in the analysis of communication and development of communication technology.

\section{B. Awareness systems and awareness-conveying functionalities}

Awareness systems "help people to effortlessly maintain awareness of each other's whereabouts and activities" [5] and are unobtrusively integrated in a user's virtual and/or nonvirtual environment. In the context of the underlying paper awareness systems or awareness-conveying functionalities are applications or functionalities of an application that enable an (automated) status message posting whereas the input and output channel of posted information is device- and/or technology-independent. Awareness functionalities (automatically) convey information on the general status (e.g. presence, feelings or demands) of a user within the application and/or between multiple applications (e.g. online, offline, idle information in IM networks). According to this definition, we like to argue that IM tools facilitating the use of awarenessconveying functionalities can be conceived as awareness systems notwithstanding these applications serve a primarily different user need. Most IM tools include a status message functionality which enables users to post ad-hoc status messages on emotional and situational states (cf. the microblogging platform Twitter). The input of status message information is device-independent since information can additionally be posted via, e.g. mobile devices or third-party applications. Skype ${ }^{\mathrm{TM}}$ and most of current IM application versions (e.g. GoogleTalk, AIM) allow the user to post status messages closely displayed to a user's name in contact/buddy lists. Furthermore a number of applications enable the information conversion from third-party applications (e.g. iTunes) to automatically generate status messages on, e.g. artist and song title information that is currently played on a device. Conclusively, Skype ${ }^{\mathrm{TM}}$ primarily is a communication tool that becomes an awareness system by enhancing the application with awareness-conveying functionalities and content.

\section{Related work on awareness systems and social presence conveying applications}

According to IJsselsteijn et al. [5] the focus of research in HCI and CSCW research shifted from pioneering examples of awareness systems which were originally developed to serve the need to stay aware of presence and availability of others within work environments to current trends in ambient intelligence originating the development of awareness systems embedded in home, work or mobile environments. Several researchers have begun to look at presence information provided by early instant messaging applications, e.g. AOL's Instant Messenger (AIM), in mobile settings and the benefits of sharing activity or location-based information.

IJsselsteijn et al. [5] report that current generations of awareness systems are all experimental and testing of applications has been conducted in mostly laboratory settings. Field tests have been limited to work environments and evaluations only considered prototypes with limited functionalities.

Bentley and Metcalf [7] provide scholars with a detailed related work description including the development and research of applications that share location information among members within social networks or that enable users sharing motion information on mobile devices. The scholars call for future research on how differently presence information can be used for the same or different purposes, on how the fusion of presence information can create richer experiences than any of the presence data taken alone or on how presence information is perceived in varying settings. Continuing, 
Bentley and Metcalf [7] see a major research gap in finding a collection of presence attributes and an appropriate presentation of these attributes that will meet the needs of users in varying settings.

Shen and Khalifa [8] investigated the design of social presence in online communities and believe that their findings on multi-conceptualization of social presence can be extended to various online social venues (e.g. IM tools).

\section{The Locatag Prototype System}

The requirements analysis was conducted following the Needs Driven Approach (NDA) introduced by Schwabe and Krcmar [9]. The concept of the LocaTag prototype system is built upon three basic premises. Firstly, a large percentage of members of a collaborative group use an IM tool, e.g. Skype $^{\mathrm{TM}}$ that supports status messages to communicate within the group, and consequently are connected to the specific IM network the majority of the collaboration-related time. As previously noted in the use case scenario the premise is a common condition in today's work environments. The second premise requires a large percentage of individuals of the same collaborative group to use an NFC-enabled mobile device, e.g. Nokia 6212 classic cellphone [10], in their everyday life. The equipment of the work environment of a group with NFC-tags comprises the third premise of the LocaTag prototype system. The NFC-tag infrastructure only needs to be established once and requires little maintenance in semi-public, indoor locations of a common work environment (e.g. company facility). Consequently it can be established by the LocaTag users themselves without requiring considerable effort.
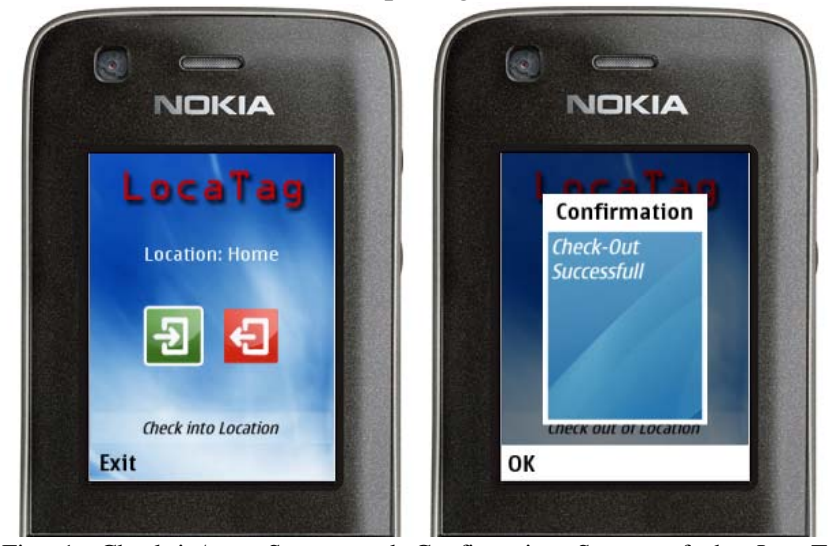

Fig. 1. Check-in/-out Screen and Confirmation Screen of the LocaTag prototype mobile client

Based on these premises, the LocaTag prototype system allows each user to check-in/-out of any location tagged in the work environment (e.g. office rooms) with their mobile phone by touching the respective tag and giving a short confirmation on the LocaTag midlet (Fig. 1).

The check-in/-out activity will then instantly be reflected by their Skype ${ }^{\mathrm{TM}}$ status message (Fig. 2), showing the updated location, provided that the user is connected to the IM network or connects with a desktop client that runs the Skype ${ }^{\mathrm{TM}}$ application and the desktop service-program of the LocaTag system.

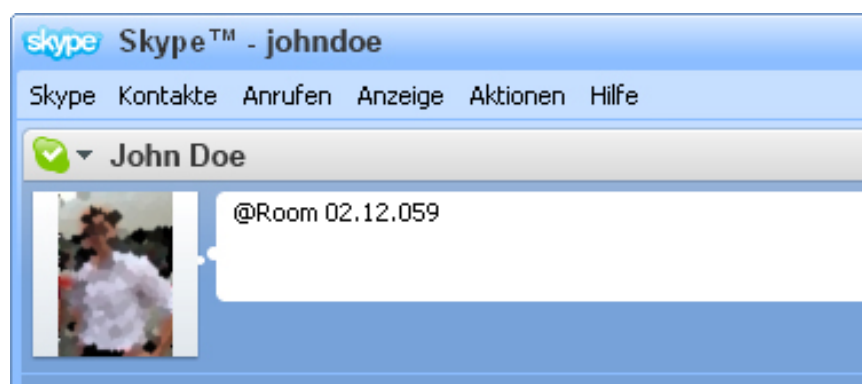

Fig. 2. Skype ${ }^{\mathrm{TM}}$ status message (mood text) created by the LocaTag prototype system

\section{TECHNICAL DESCRIPTION}

The LocaTag prototype is realized as high fidelity prototype following the Ubiquitous Computing Application Development and Evaluation Process Model (UCAN) according to Resatsch [11]. The prototype system is implemented as a distributed software system containing three distinct parts: a PHP-based webserver script, a desktop service-program and a mobile client:

1) A simple, PHP-based webserver script holding a mySQL database stores semantic locations for every LocaTag user. The user is referenced via a unique user ID (UID). The webserver allows change and information requests for the semantic location to be carried out from the other components of the LocaTag application through RESTful (i.e. Representational state transfer) HTTP queries.

2) The second part of the LocaTag prototype system is a service-program, implemented as a Java-application, running in the background on a Microsoft Windows XP desktop PC of a LocaTag user. Once the program is started it runs as a hidden thread building a mediator. On the one hand side the thread listens to the server for changes; on the other hand side the program communicates with the instance of the Skype $^{\mathrm{TM}}$ application, running on the desktop-PC through the Java API provided by Skype ${ }^{\mathrm{TM}}$ [12].

3) The third part of the LocaTag prototype system is a mobile client application, implemented on the NFCenabled Nokia 6212 classic mobile phone [10]. It is implemented as a Java J2ME midlet that can facilitate communication with the mobile phone on the one hand, and gain access to the data of the webserver component on the other hand. The communication with the webserver is done by sending and interpreting HTTP requests over the mobile internet connection. The NFC-reader device of the Nokia 6212 phone is controlled by the J2ME midlet through an API provided by Nokia [13].

The communication routine of the LocaTag prototype system (Fig. 3) can be described as follows:

In order to check-in/-out of a location, tagged with a LocaTag NFC-tag, storing a specific NFC Data Exchange Format (NDEF) record-type (urn:nfc:ext:tum:locatag), the user simply touches the tag with her NFC-enabled mobile phone. Upon touching the tag, the LocaTag midlet application 
is automatically started on the mobile device, and the semantic location, stored on the NFC-tag is transmitted to the mobile phone. The midlet then prompts the user to specify whether she wants to check-in/-out of the semantic location through a simple user interface (Fig. 2). Once the user has made her choice, a change request is made to the webserver to update the semantic location of the user with a specific UID. This UID is stored in the mobile client and desktop serviceprogram of every user. After a positive confirmation from the webserver, the LocaTag mobile application closes automatically.

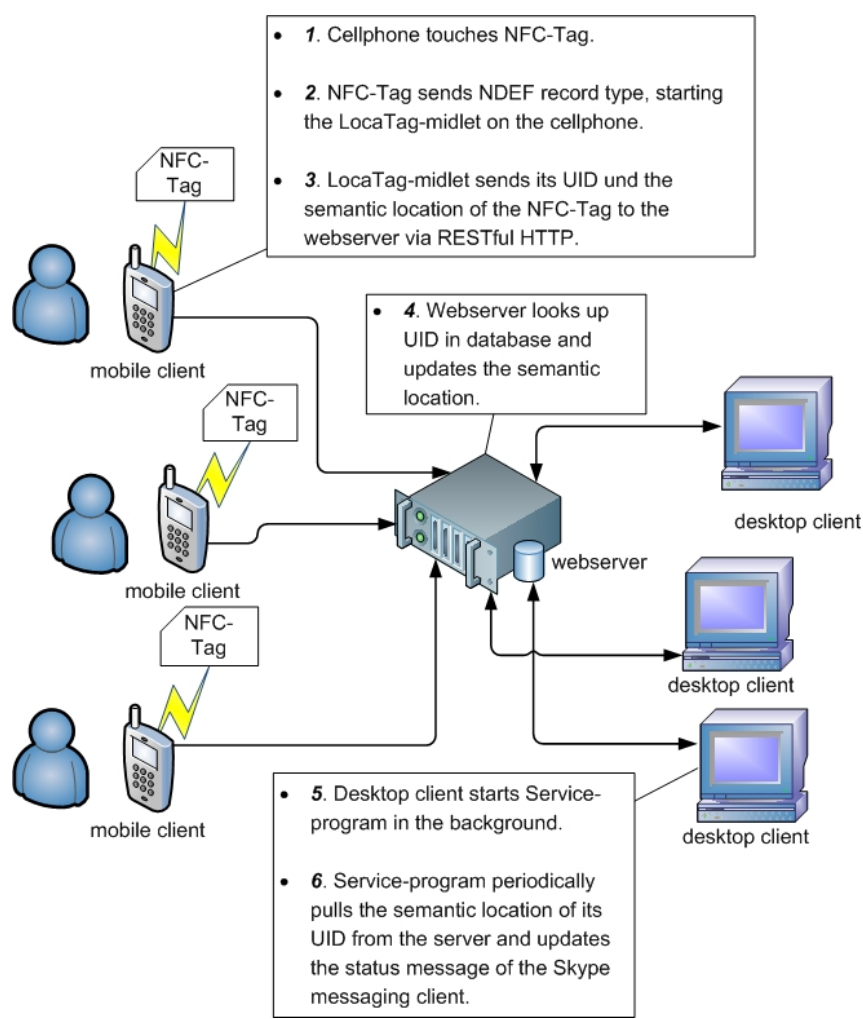

Fig. 3. Communication routine of the LocaTag prototype system (own illustration)

The desktop service-program of the LocaTag system makes a periodic information request (in the current implementation every 5 seconds) to the webserver component, inquiring the location of the user, referenced by the unique UID stored in the service-program. If the location has changed, the serviceprogram automatically updates the status message (Skype ${ }^{\mathrm{TM}}$ mood text) to reflect the change (e.g. “@ home”).

\section{Potentials AND Limitations}

The positive influence of group awareness applications and tools on collaborative work processes has been reported and hypothesized on various occasions. Liechti [3] state that "one of the major benefits of group awareness tools is to facilitate the coordination among people, and to provide cues helpful to initiate communication and collaboration". Gutwin [2] states that "group awareness is useful for coordinating actions, managing coupling, discussing tasks, anticipating others' actions, and finding help”. Oulasvirta et al. [4] in their analysis of "awareness cues" in three field trials report that the cues "place, proximity, movement, and activity" were relied upon when initiating (face-to-face) communication within collaborative groups. It is therefore likely, that the nonintrusive, almost ambient integration of presence and location information into an existing and commonly used IM tool through the use of status messages could provide useful awareness cues to its users and increase the coordination and initiation of communication and collaboration processes within a group. An NFC-based check-in/-out routine, as described in this paper is one of the most convenient ways imaginable to provide the required location information.

The paper demonstrates the technical feasibility and potentials of a combination of commonly used IM systems and location information retrieved from an NFC-based check-in/-out process. While the current implementation of the LocaTag prototype system is functional for exemplary use scenarios, we want to allude to some systemic flaws of the current version. At the moment, the LocaTag system provides only real-time location information as long as the user is connected with her Skype $^{\mathrm{TM}}$ application on a desktop Windows PC. Consequently the LocaTag user might still check-in/-out of a location by touching the NFC-tags while her Skype ${ }^{\mathrm{TM}}$ application is not connected with the Skype ${ }^{\mathrm{TM}}$ network (offline status), but the location update will not be available to her peers until she changes from an offline to an online status. The described design issue is due to the fact that the Skype ${ }^{\mathrm{TM}}$ application does not support a change of status messages unless the user is logged in and in the online status. However, we want to argue that a user who is offline in the Skype ${ }^{\mathrm{TM}}$ application is not in the center of attention of her online peers, and thus, a location update of an offline user would only be noticed by a minority of peers anyhow. With advances in mobile device technology (e.g. smartphones) and a general increase in mobile internet connectivity, the LocaTag system could be completely integrated into mobile devices. However, there is no mobile device currently available on the market that features the technical requirements to run a mobile Skype ${ }^{\mathrm{TM}}$ or similar IM applications and which is equipped with an integrated NFCantenna.

Privacy concerns of users, especially in work environments, might hinder usage and adoption of systems and applications similar to the presented LocaTag concept. The implication of information on a user's current location during work hours or on stored past locations, made available to a superior, is likely to raise privacy concerns. However, in contrast to a system that derives location information through a Global Positioning System (GPS) receiver, and automatically updates a status message without the user's active participation or knowledge, a location update with the LocaTag system has to be initiated by the users themselves by establishing a near physical contact between mobile devices and an NFC-tag. Since the user has full control over this process, the LocaTag system might not be perceived as a threat towards privacy to a larger extent. Yet this assumption needs to be verified in a long-term quantitative field study. 


\section{FUTURE RESEARCH PROSPECTS}

So far, the status of the LocaTag implementation is that of a proof of technology prototype.

Therefore a planned long-term field study should deliver insights on possible effects of frequent system usage and/or adoption on group awareness and connectedness. Additionally a long-term field study would also enable the assessment of the general usability of the system and evaluation of practical issues, e.g. the composition of status messages. Therefore further questions on the design and conveyance of status messages within awareness systems could be tested: "What kind and granularity of location information is important to the virtual community/group?" "Should there be different location information shown for different contact groups, e.g. the description '@Work' for friends and '@Room \#' for coworkers?’

From a technical perspective, the LocaTag prototype system could be enhanced with the use of GPS-receivers embedded within mobile devices. In that case, locations would not have to be physically tagged by the user, but virtually defined as a radius around a GPS-coordinate. The mobile application of the LocaTag system could then prompt users whenever she enters or leaves a new or existing location, whether she wants to check in-/-out. While the use of GPS-technology would eliminate the momentarily limiting requirement of NFCenabled mobile devices, it holds some technical drawbacks, e.g. the limited reception of a GPS-signal in buildings or the need for a constant sensing of the GPS-position.

Use case scenarios other than work-related environments for similar applications are imaginable, e.g. tracking of patients or checking a patient's health status by relatives.

\section{REFERENCES}

[1] A. F. Cameron, and J. Webster, "Unintended consequences of emerging communication technologies: Instant Messaging in the workplace," Computers in Human Behavior, vol. 21, no. 1, pp. 85103, 2005.

[2] C. Gutwin, R. Penner, and K. Schneider, "Group awareness in distributed software development." in Proceedings of the 2004 ACM conference on Computer supported cooperative work. Chicago, Illinois, USA, 2004, pp. 72-81.

[3] O. Liechti, “Awareness and the WWW: an overview," SIGGROUP Bull., vol. 21, no. 3, pp. 3-12, 2000.

[4] A. Oulasvirta, R. Petit, M. Raento, and S. Tiitta, "Interpreting and acting on mobile awareness cues," Hum.-Comput. Interact., vol. 22, no. 1, pp. 97-135, 2007.

[5] W. IJsselsteijn, J. van Baren, and F. van Lanen, "Staying in touch: Social presence and connectedness through synchronous and asynchronous communication media," in Proceedings of the 10th International Conference on Human-Computer Interaction. 2003, pp. 924-928.

[6] R. Rettie, "Connectedness, awareness and social presence," in Proceedings of the 6th Annual International Workshop on Presence, Aalborg, Denmark, 2003, pp. 6-8.

[7] F. Bentley, and C. Metclaf, "Sharing motion information with close family and friends," in Proceedings of the SIGCHI Conference on Human Factors in Computing Systems. San Jose, California, USA, 2007, pp. 1361-1370.

[8] K. N. Shen, and M. Khalifa, "Design for social presence in online communities: A multidimensional approach,” AIS Transactions on Human-Computer Interaction, vol. 1, no. 2, pp. 33-54, 2009.

[9] G. Schwabe, and H. Krcmar, "Piloting Socio-Technical Innovation," in Proceedings of the 8th European Conference on Information Systems, Vienna, Austria, 2000, pp. 132-139. http://europe.nokia.com/find-products/devices/nokia-6212-classic/. F. Resatsch, "Developing and Evaluating Ubiquitous Computing Applications Using the Example of Near Field Communication in Germany,” Dissertation, Chair for Information Systems, Technische Universität München, München, 2009.

[12] Skype ${ }^{\mathrm{TM}}$. "wiki/Java API - Skype Developer Zone," 12.12.2009, 2009; https://developer.skype.com/wiki/Java_API.

[13] Nokia. "Forum Nokia - Near Field Communication," 12.12.2009, 2009;

http://www.forum.nokia.com/Technology_Topics/Mobile_Technol ogies/Near_Field_Communication/. 